\title{
ON SOME AFFECTIONS
}

\section{OP THE \\ CECAL PORTION OF THE INTESTINES.}

\author{
WITH ILLUSTRATIVE CASES.
}

BY

FREDERICK GEORGE REED, M.D., M.R.C.P.

Received Dec. 10th, 1861-Read Jan. 14th, 1862.

THe interest which usually attaches to diseases occurring within the cavity of the abdomen induces me to think that the relation of a few cases illustrative of one form of disease, of not very infrequent occurrence, will, in some degree, be conducive to the objects of the Society, and, under that impression, I beg to present them to the notice of its Fellows, with a few prefatory observations.

In speaking, generally, of the diseases of the intestinal tube, we recognise two conditions-the one, resulting from an excessive accumulation of intestinal contents or the impaction of foreign substances, at some particular part of its course, as its primary cause ; the other, as commencing in the structure of the intestine itself, apparently independent of any accumulation or lodgment; and to these I might add a third, as resulting either from visceral displacements, or from formations that take place exterior to the intestinal tunics, but occasionally influencing the diameter of its canal and its functions.

These several conditions may lead to very similar consequences, so that in the progress of a case there may be an 
occasional difficulty in deciding upon which of the conditions mentioned the existing symptoms depend. It is, however, usually otherwise, and the symptoms which present themselves, if considered both with regard to the manner and rapidity of their development, render their recognition not very difficult, particularly with respect to the two conditions first mentioned.

The four subjoined cases, I presume, may be considered as referable to the first-named condition, viz., " to accumulation or lodgment of the intestinal contents in the cæcum and commencement of the colon;" for circumstances suffciently conclusive are mentioned in the relation of each, to raise the strongest presumption of the correctness of this view. Now, as this condition is the most favorable for securing relief to the patient by proper treatment in its early stages, it becomes a matter of extreme importance to have the means of recognising it before it has passed into its more severe and dangerous stages.

Having this in view, it may be stated, generally, that the symptoms in these conditions are not infrequently (indeed, it is probable, are most frequently) developed with greater rapidity and partake less of a chronic character than in obstructions resulting from changes in the structure of the intestine itself; and although the observation may be of little practical value, it should not be lost sight of, either in the diagnosis or in the treatment of these cases. The symptoms, however, which are of real value in determining the diagnosis of these cases are of a local character, and are the direct consequences of the accumulation within the intestine. As might be anticipated, there is a local tumour, dull on percussion, as the result of excessive accumulation, varying in size and form, and which some of the cases show is well defined, and is better examined whilst the patient is recumbent on the back, with the knees bent. This tumour is more or less painful on being firmly pressed with the hand, and as the symptoms advance the pain extends to the neighbouring parts of the abdomen, and eventually the whole abdominal cavity may become involved. 
It should be borne in mind that the existence of local pain without the coexistence of other symptoms is insufficient for the object in view, for in obstructions of intestine which occur so frequently on the left iliac region, in the sigmoid flexure of the colon, the pain is often referred to the right side and to the region of the cæcum, and frequently also to the umbilicus. The pain, when referable to this cause, is, however, not aggravated to any great extent by pressure, neither does it radiate from the cæcum over the abdomen, as from a centre.

Taking these symptoms in connexion with those circumstances (which, probably, may be elicited by inquiry) with respect to the taking of indigestible substances into the stomach at no distant period of time antecedent to the symptoms, and having ascertained the previous habit and condition of the patient (particularly is this necessary in gouty or asthenic cases, from the liability in these subjects to attacks of colic) and specially in relation to the imperfect mastication of food, habitual constipation, or the passage of dry, hardened fæces from time to time, there will generally be no great difficulty in arriving at a just conclusion as to the nature of any case of a similar kind to the subjoined; and having done so, the proper general treatment to be pursued for the relief of the patient becomes a question of easy solution. It must be borne in mind, however, that the intensity of symptoms becomes greatly varied as cases of this kind proceed in their course, leading from those of simple distension of the intestine to the severest forms of inflammation and its consequences. The treatment which would be suitable for the first state would not be suitable for the other states.

Thus, in the early stage, when distension only exists, the obvious remedy is to unload the distended part by some means, and if this be done effectually the impending inflammatory stage is not developed. This would appear to be most certainly accomplished by the use of the hot-water hip-bath, followed by fomentations, and large emollient cataplasms applied over the abdomen, and also by injections 
per anum of warm water or medicated injections, according to the features of each case, accompanied by the administration of such medicines as are known to stimulate the action of that part of the intestine in which accumulation occurs; and, perhaps, the most suitable medicine for this purpose is that preparation of aloes known as its "watery extract," administered in small and frequently repeated doses, in the form of pills, with soap (for an adult, one-grain doses of the extract with two or three grains of soap), given every hour, and, under certain circumstances, it may be combined with calomel, or, in asthenic cases, with the extract of nux vomica, if not contra-indicated. Should there be nausea or the stomach be irritable, frequently repeated doses of saline effervescing medicines, with an excess of alkali, may also be given with advantage. When, however, a case has passed into its inflammatory stages there should be a corresponding change of treatment, or, rather, an addition to the formernamed treatment, suitable to the relief of inflammation, as detailed in the cases brought by me under the notice of the Society. Again, the inflammation may lead to its usual consequences, provided the patient does not sink directly under its influence.

These consequences are well illustrated by the following cases. In the first, the patient did not survive the inflammatory stage. In the second, an abscess was established, and a fistulous opening of communication with the intestine formed, but it fortunately closed at a subsequent period under a treatment recommended by Sir Benjamin Brodie. In the third, mortification of the intestine occurred to a very great extent, and most remarkable powers of reparation and recovery were brought into play.

It would be misplaced whilst offering these observations to the Fellows of this Society, for me to enter either upon the remarkable anatomical formation of the cæcum, with its occasional variations, its peculiar physiological functions, or upon the diagnostic signs of the several abnormal conditions with which accumulation or lodgment, followed by obstruction in the cæcal portion of the intestinal canal, may possibly be 
confounded, and which render the special affections of this viscus peculiarly interesting.

I trust, in the narration of the accompanying cases, many of the prominent practical points are sufficiently indicated, without unnecessary prolixity.

Case 1.-On 13th August, 1858, Mr. Nowell Stowers, of Newington Place, Kennington, was requested to see A. B-, æt. 18, the son of a gentleman residing at Brixton, Surrey, who previously had enjoyed perfect health without any exception. He was short in stature, of a sanguine temperament, and of a robust and strong constitution.

Mr. Stowers stated that he found him suffering intense pain in the abdomen, of a colicky nature, and with tenderness on pressure in the right inguinal region; his skin was hot, his pulse quick and full, and tongue furred. He had no sickness.

He had been in his usual good health, and taking daily active horse-exercise, up to two days previously, when he had eaten a considerable number of unripe plums and largely of lobster salad. During the course of the same night and following morning, feeling, as he stated, "uncomfortable in his interior," and his bowels being constipated, he took a table-spoonful of castor oil, which he repeated after a time. As neither of these produced any effect, he procured from a chemist in the neighbourhood, and took, a calomel pill and black draught. These produced a very violent effect, acting twenty times at least, attended by violent tenesmus, dislodging a large fæcal accumulation and a great many plumstones.

Each evacuation was attended by considerable pain in the abdomen, and was followed by great feeling of exhaustion.

The pain in the bowels having increased, contrary to his expectations, Mr. Stowers was sent for. He immediately prescribed leeches to the right side of the abdomen, to be followed by warm fomentations, and also small doses of calomel and opium, and carminative and astringent medicines, to relieve the diarrhœa and tenesmus.

vol. XLv. 
I was requested on the evening of the 14th August to see him, in consultation with Mr. Stowers.

On my arrival I found the patient was suffering great and constant pain, extending over the whole of the abdomen, which was increased on deep pressure, but especially felt in the right iliac and inguinal region, extending into the right hypogastric and the umbilical regions. There was a distinctly defined swelling, about the size of a small orange, in the situation of the cæcum. It was dull on percussion, and the pain was most intense in this part on coughing, deep inspiration, and on extending the right thigh. There was not any sickness. His pulse was hard, and 96 in the minute ; his skin hot, but not remarkably so. His countenance had rather an anxious expression. His tongue was covered by a yellow coating, and its edyes were rather dry. There was thirst, but not excessive. The breathing was thoracic, the urine turbid, and loaded with lithates, sufficient in quantity, and free from albumen. $\mathrm{He}$ had constant tenesmus, voiding only small quantities of mucus, tinged with blood.

He was ordered leeches to the right iliac region, one grain of calomel, and one third grain of opium every hour ; turpentine stupe, succeeded by linseed poultices, to be applied to the whole of the right side of the abdomen; an opiate suppository, and perfect rest and farinaceous diet; and all solids were prohibited.

On the following and subsequent three days there was but little or no alteration or improvement in his general symptoms; but the tumefaction in the right iliac region increased considerably in size, though not in tenderness; neither were the symptoms of general peritonitis more decided.

I saw our patient twice daily in consultation with Mr. Stowers, and the same plan was continued, but the doses of calomel and opium were more frequently administered. On the 18th a peculiar fœtor in his breath was noticed; his countenance became more anxious, and he became more feeble, and he had occasional hiccup, and some tympanitis existed; his bowels acted frequently and involuntarily, with 
small quantities of liquid muco-feculent evacuation, tinged with blood, and very offensive.

His pulse became soft, weak, and compressible; his voice feeble ; there was still total absence of nausea, eructation, or sickness; his skin was cold, moist, and clammy; there was no shivering, and no increase or remarkable diminution of pain, and no head symptoms ; but the aspect of the case could not be well more unpromising.

The gums showed evidence of slight mercurial action, the calomel therefore was forthwith discontinued, and he was ordered increased quantities of strong animal broths and port wine, \&c.

On the 19th the evidence of mercurial action was more fully marked, and from this date his symptoms gradually diminished in intensity, and his strength appeared to improve, but a tender and solid swelling, of about four inches in circumference, which had become distinctly circumscribed, remained on the right side of the abdomen, principally in the right iliac and inguinal regions.

Occasional doses of grey powder, with a view to maintain its action, were subsequently administered. Blisters, iodine, and mercurial ointments were applied locally. He was directed to take plenty of liquid, animal, and farinaceous nourishment, with wine daily, but no solids of any kind. Under this treatment his improvement continued gradual and satisfactory up to the 2nd September (three weeks from the commencement of his illness). At this time the general abdominal tenderness had nearly disappeared, but the hardness and fulness of the cæcal region continued, with slight tenderness in the part, and a sensation of internal soreness, though the swelling had considerably diminished in size.

The diarrhœa and tenesmus which continued were kept in check by opiate suppositories and acetate of lead, sulphate of copper, and gallic acid, which, from time to time, were administered internally up to the 2nd of September. On this day he was so well that he was allowed to sit up in a chair for a short time. Late in the evening of this day 
the nurse discovered, after an action of his bowels, that he had passed something extraordinary, but without his personal knowledge, or causing him any unusual sensation. Mr. Stowers was immediately sent for, and on examination he found it apparently to be a portion of intestine, ten to twelve inches in length, surrounded by a large quantity of dark matter, with portions of fæcal matter adherent, and mixed with blood and mucus voided at the same time.

The patient was forthwith ordered to bed again, to be kept quiet, and in a recumbent position, and to avoid all exertion, especially straining or making any effort at stool. The bowels became, however, very irritable, and he passed frequently liquid fæcal motions, mixed with dark, bloody mucus ; this symptom was treated by opiates and astringents, and he was restricted still to animal broths, with rice, arrowroot, \&c. After this he cortinued to improve generally, without any remarkable new symptom up to the 10th October, when he was so well that we deemed it advisable he should go to Brighton for sea air.

While there, he had strict injunctions to continue the same diet, and to avoid all solid food for some weeks, and gradually to take an increase of exercise. He was also ordered to take the tincture of sesquichloride of iron in ten to twenty minim doses, two or three times a day, and to keep the diarrhœa under control by occasional doses of acetate of lead, or gallic acid with opium, and to take a dose of castor oil if the bowels were not satisfactorily acted upon daily. This constituted his treatment for three or four weeks, after which time he was permitted very gradually to return to his ordinary diet and habits; and, except suffering from occasional looseness in the bowels, and pain (on any over exertion) in the right iliac region, he continued to improve for several months, until all uncomfortable sensations disappeared, and he felt quite well.

He has for the last eighteen months been, and is at the present date, (December, 1861), after the lapse of more than three years from the commencement of his illness, in perfect 
health in every respect, with no perceptible alteration in any function or sensation.

Accompanying this paper, is the mass voided, which was given to my late friend Mr. Quekett, for the museum of the Royal College of Surgeons. Mr. Quekett kindly examined it minutely with much care and interest, and the following is his report upon the subject :

"I find that the preparation submitted to me, consists of portions of the intestinal canal, the parts best marked being the cæcum and appendix vermiformis. As now mounted, the mucous membrane of the cæcum may be seen at the upper part, and that of the appendix at the lower part of the preparation.

"When examined microscopically, both these structures exhibit the follicles of Lieberkühn, and the absence of villi. The muscular, and also the peritoneal coats, are rery apparent in some parts, and agree in all respects with those of ordinary healthy specimens of these tissues. The length of the specimen, as it is now mounted, is between nine and ten inches ; and I should consider it a portion of the large intestine, with cæcum and appendix vermiformis. Of the former, only about one third of the calibre of the canal, whilst of the latter the entire tube was present. We have nothing of the kind in the museum of the College of Surgeons, and this circumstance alone would induce me to believe the occurrence was one of considerable rarity."

Instances of recovery, after portions of the intestinal canal have been passed per anum, with the cylinder entire, have been recorded; but peculiar interest is attached to the present case, as I think it may be assumed (after careful examination of the preparation exhibited) that intussusception did not exist, but that, from the enteric inflammation and its results (originatiug in the cæcum), it is not improbable that the anterior surface of the large intestine voided became agglutinated to the peritoneal surface of the abdominal parietes, and that the portion, or "section" it might be termed, of the intestinal tube, with the cæcum and 
appendix vermiformis, were thrown off by ulceration, and subsequently passed per anum; the void having been repaired, and the continuity of the canal maintained by the adaptation of the effused lymph to the parietes of the abdomen, the adhesions formed having also prevented the escape of any of the contents of the bowels into the peritoneal cavity.

Case 2.-On the 13th April, 1846, I was requested to see A. T-, æt. 14, a fine, tall, well-developed girl. I learnt at my first visit she had been seized suddenly with pain in her abdomen the day preceding. Her bowels having been constipated for some days previously, she had in consequence taken several pills and other aperient medicine, which, however, had produced no action of the bowels up to the time of my seeing her, but the pain was stated to have gradually increased. She had not previously eaten anything unusual, neither could the attack satisfactorily be accounted for, but was supposed to arise from cold.

I found the abdomen rather distended, and she complained of pain on slight pressure, increased on doing so firmly. It appeared to be most severe in the right iliac fossa, but I could detect only a slight fulness, but no hardness or dulness in that situation. She had a feeling of nausea, but had not vomited. Her skin was cool and moist, her pulse sixty-five in the minute, small, and compressible. There was no hernia. Her countenance was rather anxious, and she said " her feelings of illness told her she should die." I ascertained she was a nervous, sensitive girl when she did not feel well, and was very hysterical, which circumstance rather obscured the diagnosis. I ordered her a warm hip-bath, followed by warm linseed poultices to the right iliac region, a full dose of calomel and opium, and a turpentine enema.

The day following I found the bowels had acted twice after the injection. The evacuations were stated to have been healthy and frcal, though hard and constipated; but the pain in the abdomen had considerably increased, especially in the right iliac fossa, where a distinct fulness, about three 
inches in extent, dull on percussion, was distinguishable. She had vomited several times, and I entertained no doubt that inflammation existed. Leeches were applied, followed by a turpentine stupe and half a grain of calomel, and one eighth grain of opium, was ordered to be taken every hour, and fluid nourishment only, and quietude.

The next day the local pain had increased, but not the swelling; all her general symptoms had become more severe, tympanitis existed, and there was continued vomiting. There had been no action of the bowels since the effect of the first turpentine injection.

On the morning of the 4th day she appeared approaching a state of collapse. I directed the calomel and opium which had been given up to this time to be discontinued, and brandy and wine, with soda water, to be given ad libitum.

On the morning of the 5th day she expressed herself as much easier, and "thought something had given away in her bowels, which was the cause of her relief," but she appeared in a sinking state. There was much less fulness in the right iliac region, and less abdominal tenderness.

The day following (the 6th), I found she had rallied considerably, and that she had been able to take stimulants and nourishment, but diarrhœa had set in, offensive liquid fæcal matter passing continually, mixed with blood and mucus.

This continued for three or four days, being only partially checked by various astringents with opium. The vomiting having, however, ceased, she was able to take nourishment and stimulants, and her strength improved.

On the 11th day a solid mass passed from her bowels without causing her any pain or discomfort, which I found on examination to be six or seven inches of intestine, in a gangrenous state (apparently large intestine). I ascertained that the calibre of the canal was complete, and it was surrounded by some dark bloody mucus. She continued to improve from this date, but suffered from diarrhœa and dysenteric symptoms for five or six weeks, attacks of which became, on two or three occasions, so severe, and lasted from twenty-four to thirty-six hours, producing so much 
depression and exhaustion that it appeared probable she would sink from this cause.

Lead, bismuth, and many mineral and vegetable astringent medicines combined with opium, were prescribed, but sulphate of copper appeared to be the only medicine that exerted any lasting beneficial influence. It was given in doses of one twelfth of a grain, with one eighth of a grain of opium, in the form of pills, every three hours, for three or four consecutive dass, when the dysenteric symptoms had nearly ceased. The diarrhœa recurred from time to time, but was always controlled by a few doses of the same medicine. She was restricted to fluid nourishment only, until about the expiration of two months from her first seizure, when all symptoms of her illness had disappeared, and she returned to her former habits.

She continued quite well, only suffering from hysteria and habitual constipation for seven or eight years subsequently, and is alive and well at the present time (December, 1861).

The morbid specimen was unfortunately thrown away by one of her family, after I had placed it aside for preservation.

CASE 3.-A. B., æt. 20, a stable groom, a very healthy young man, strong, robust, active, and of sanguine temperament.

Arose from his bed at 5 a.m., feeling quite well. Shortly afterwards he ate hastily three or four good-sized apples, not fully ripe. He subsequently performed his accustomed stable work and rode a saddle horse for exercise for an hour.

At 8 a.m. of the same day, he made a hearty breakfast of meat.

At 9.30 a.m., whilst grooming a horse, he was suddenly seized with intense pain in the right iliac region, which obliged him to go to his bed. He was advised and took a dose of castor oil, and, subsequently, some tincture of rhubarb, which acted two or three times freely it was stated. 
I was requested by his master to see him a few hours afterwards. He was lying on his back, with his right thigh bent, and complained of severe pain in the right iliac fossa, in the situation of the cæcum, extending in the direction of the ascending colon. There was no general peritoneal tenderness or any local tumour or swelling, but the pain was much increased on firm pressure. He had vomited several times, and it was stated that the breakfast he had eaten had returned, and he said "he felt convinced some hard apples he had stuck there" and that he could put his finger on the most painful spot in the right iliac region. His bowels had acted freely two hours before I saw him. There was no hernia. His countenance and manner were very anxious and troubled; his skin was moist and clammy; his pulse quick and full.

I ordered him a warm hip-bath, to be bled to fourteen ounces, twenty leeches to be applied to the painful part, succeeded by warm emollient poultices. A full dose of opium to be taken directly, and followed every hour by onegrain doses of calomel and one third of a grain of opium; no further aperient to be given, and fluid nourishment only.

Late in the evening of the same day I found diffused peritonitis had set in. A turpentine stupe was applied externally over the whole of the right side of the abdomen, and a gruel injection with turpentine was ordered, which was followed by two healthy fæcal evacuations from the bowels, and produced some feeling of relief.

On the 3rd (the following day), in the morning, his inflammatory symptoms had become more severe. Tympanitis had come on, and the pain occurred in paroxysms, and he appeared to suffer great agony. Opiates in full doses gave only slight relief. Vomiting occurred occasionally, but there was no fæcal smell in the matters ejected. Nothing further had passed from the bowels.

The calomel and opium and other measures were ordered to be continued. On the night of the third day the symp- 
toms of collapse appeared, and he sank early in the morning of the fourth day.

On inspection the following day, the peritoneal cavity was full of bloody serum, with flakes of lymph. The convolutions of the small intestines were all highly gorged and glued together, in many parts, by recent adhesive matter, and they were also distended with gas, and contained a considerable quantity of pulpy matter.

The cæcum (which appeared to have been loosely connected congenitally by peritoneum to the right iliac fossa) together with the appendix vermiformis and about four inches of intestine which I took to be ileum, thickened and surrounded with masses of bloody lymph, were invaginated within the ascending colon, and appeared in a partial state of sphacelus. On carefully examining the interior of the cæcum, a portion of the core of an apple, about one third of an inch in diameter, was entangled, and appeared stuck in the free edges of the cæcal portion of the ileo-colic valve, and required some slight effort to remove it with forceps from its bed. All the structures of the transverse and descending colon were much thickened and surrounded by dark coloured lymph from inflammatory action. The stomach and duodenum and rectum were healthy.

Having promised his relatives that no portion of the nbdominal contents should be removed from the body, if a post-mortem examination was allowed, I was not permitted to bring away the very interesting morbid specimen. The case was seen by me in consultation with Dr. Furnival, who was also present at the post-mortem.

CAse IV.-A lady, æt. 23, short in stature, of an unusually spare habit of body and leucophlegmatic temperament, and very fragile in appearance, but who had always enjoyed perfect health. Shortly after her marriage, in the latter part of the year 1848, became pregnant, not suffering more than the ordinary discomforts of that state. At the end of July, 1849, she was prematurely confined at the seventh 
month, the child being in a state of putrefaction. The cause of this miscarriage was supposed to have been an accidental fall. She had considerable post-partum hæmorrhage, but she recovered without any other unfavorable symptom. She had, however, suffered from mental depression and debility consequent on the disappointment at not having a living child, and the loss of blood she had sustained. Otherwise she felt, and was considered by her friends to be in her usual good health, until the 16th or 17th of November in the same year; she was then suddenly seized with pains in her bowels. A medical gentleman residing in the neighbourhood being consulted, leeches were applied to the abdomen, and some laxative and sedative medicines were administered. I was requested to see her in the evening of the same day. The following was her condition at my first visit :

Her countenance was flushed, but not anxious. Her skin was hot and dry; her pulse was small, hard, and not readily compressed; her tongue was moist and flabby, with a slight yellow coating over the whole surface. She had great pain in her bowels, and was fearful about the result. She complained of nausea, and had retched several times, but had not vomited.

On putting my hand on the abdomen there was found to be a general tenderness, but the pain was most intense in the right iliac region, where there was considerable and diffused tumefaction, and a tumour about the size of the double fist of an ordinary man situated deep in the fossa, with a defined margin, and dulness on percussion was also detected. I satisfied myself there was no hernia. The abdomen generally appeared full, but not tympanitic. There was no nephritic pain or tenderness, but the urine was loaded with lithates.

On examination by the vagina, the tumour could with some difficulty be reached by the finger; it had a doughy feel, was round in form, and painful on pressure. I could, however, feel the right ovary distinctly, much larger than ordinary, but not tender; the uterus -was natural and healthy. On close inquiry it was elicited that the bowels 
had been very constipated for several days, and that for some time previously they had always acted scantily and with difficulty, and chiefly small hard lumps passing.

She thought her illness had "something to do with her confinement, and could not say how long the swelling had existed, as she had never noticed it."

I thought the tumefaction arose from some impaction in the cæcum and that enteric inflammation had been set up with diffused peritonitis; I accordingly ordered leeches to the right side, succeeded by turpentine stupe.

Calomel, gr. j, opium, gr. $\frac{1}{3}$, every hour. A large warm linseed poultice over the whole abdomen, and exclusively fluid farinaceous diet.

On the 18th (the day following) the pain had slightly diminished, her bowels had not acted. I ordered an enema, with turpentine and gruel, three pints at least, which acted three or four times, dislodging a considerable quantity of hardened feculent matter, and the previous treatment to be continued.

On the third day (19th), I was disappointed in finding that no improvement in the symptoms had taken place from the free action of the bowels. On the contrary, the swelling in the right iliac region had increased; the pain was more intense and diffused, extending into the right hypochondriac region. The patient could not bear any movement. The stomach had become irritable with constant nausea and retching, and she also complained of most intense pain in the right hip and dorsum of the ilium, extending down the thigh, leg, and foot, in the course of the anterior crural nerve.

I directed the same doses of calomel and opium to be given every half hour till the gums became affected; saline effervescing draughts, (with an excess of alkali) and hydrocyanic acid, every three or four hours, to allay the sickness; also croton oil, to be applied externally over the whole of the right side of the abdomen, and covered with warm poultices, and another large turpentine enema to be used, and repeated in three hours, if an action of the bowels did 
not take place. The first produced two or three very offensive liquid fæcal evacuations.

From the 19th to the 23rd she continued in a variable though unsatisfactory state. Although all her symptoms were subdued in intensity, and the pain in the right side of the abdomen had become a much less prominent symptom, she was yet unable to bear any pressure or movement. The irritability of the stomach, however, had ceased, and she was able to take animal nourishment in a fluid state, and also by enemata, combined with starch. The calomel and opium were continued, though the quantity and intervals were varied, according to the daily, almost hourly, intensity of the pain and symptoms; and her strength was supported generally by nourishing diet in a fluid state. Her pulse varied in some degree; her tongue and her several symptoms presented no remarkable variation of interest worthy of being mentioned, except that the pain became more localised in the right iliac region; the swelling gradually increased, extending into the umbilical and right hypogastric region.

There was also increasing and most intense pain in the hip, dorsum of the ilium, and groin; also in the course of the anterior crural nerve of that side; and the right thigh, leg, and foot, gradually became oedematous, pitting deeply on pressure. In fact, the patient complained of this more than any other symptom, and continually exclaimed, "All her disease arose from her hip-joint." Opiates were administered internally in large doses, and belladonna with camphor applied externally with relief, and the whole limb was enveloped in wool.

On the 24th of November the mercury was entirely discontinued, and opiates in various doses and forms were given to relieve the pain in the hip and right leg.

On the 28th of November, Sir Charles Locock met me in consultation, and subsequently on the 3rd of December Dr. Robert Ferguson's assistance was also requested. An opinion was at first entertained that an enlarged ovary might have become inflamed, and thus pressing on a portion of the bowel, might have caused obstruction; but the exami- 
nation by the vagina decided this not to be the case; the rectum was found to be quite empty, and the right side of the pelvis appeared blocked up with a large hard mass. It was determined to pursue the same plan of treatment, and to solicit the bowels to act by daily injections of warm gruel only.

From this date to the 7th of December no material alteration took place, except that the strength of the patient decreased, but her stomach remained quiet, and thus enabled her to take considerable quantities of strong animal broths, wine, brandy, \&c.

From the 7th to the 10th, symptoms of collapse came on, with hiccup and occasional fits of shivering, and the swelling became externally much more prominent, followed, in a day or two, by redness and a tendency to point just above Poupart's ligament and mid space between the anterior inferior spinous process of the ileum and the linea alba.

She appeared to rally under the very large quantities of nourishment and stimulants she was able to take; and as she had not appeared to have any satisfactory relief from her upper bowels for nearly three weeks, a strong turpentine injection, combined with ox-gall, with warm gruel, in all upwards of two quarts, was carefully and gradually administered. This was followed, in half an hour, by an enormous discharge of nearly two chamber-potsful of the most offensive fæcal matter. Extreme prostration and threatened sinking followed, and continued for twenty-four hours more, and she appeared on the point of death, but she again rallied under the use of large quantities of brandy and ammonia.

Two days after this occurrence there was a great feeling of relief, and the tumour had diminished considerably ; but fluctuation was now perceptible externally, and it became evident that an abscess had formed. The abdomen was soft, and of its natural size in other parts, and free from pain, but with slight tenderness on pressure.

The pain in the hip, leg, and foot entirely disappeared, and the swelling also subsided gradually from this time.

On the 20th of December, nearly five weeks from the 
commencement, the abscess burst externally; ten or twelve ounces of most offensive, dark-coloured pus were discharged, and subsequently small portions of fæcal matter began to ooze out with the pus. Warm fomentations were used, and linseed poultices were applied externally. Two or three days following it was discovered that pus in considerable quantity, mixed with fæcal matter, passed from the bladder each time the patient voided urine, which was every hour or two.

The bowels became irritable; the patient exhausted and hectic; and her state fluctuated for two or three weeks; but, by the aid of nourishment, and the continuous use of stimulants, opiates, and tonic medicines, notwithstanding two to four ounces of pus passed daily from the wound, she gradually gained strength.

The communication with the bladder appeared to be healed spontaneously; the bowels acted healthily, though very scantily, with the aid of daily iujections of warm water, no aperient medicine being given by the mouth; but a fæcal fistula became established where the abscess burst externally in the inguinal region, and, according to the excited or quiescent peristaltic movement of the bowels, fæcal matter passed constantly, varying in quantity though daily much more considerable than that passed per anum. The opening was about half an inch in length, oval in form, with slightly ulcerated, elevated, irritable-looking, and everted granular edges. A probe could be introduced three or four inches, and moved to and fro, apparently in the cavity of the cæcum. There was constantly a most offensive odour from the wound, and the matter passing was peculiarly offensive and sickening.

The patient appeared to have recovered her health generally, but any movement or exertion gave her great pain in the region of the cæcum. She had ceased to menstruate from the commencement of her illness up to this time.

On the 24th of June, 1851, seven or eight months from the attack, Sir Benjamin Brodie saw the case in consultation 
with me, and was much interested with its details and nature.

He gave a doubtful prognosis as to complete recovery, but advised her having a couch made so as to be recumbent on her face day and night for several months; to have the edges of the wound touched occasionally with nitrate of silver, and to take $3 j$ doses of powdered cubebs twice daily for some months, and of course to adopt all general measures for the improvement of her health, and the occasional use of injections per anum if necessary. The result proved most gratifying and successful. The patient most rigidly and faithfully maintained the position recommended both by day and by night, and after a few days stated "that instead of finding the position irksome, it afforded her such a feeling of local relief and also comfort from the diminution of the discharge, that she should be most unwilling to alter it until quite well." At the end of six months, the discharge ceased entirely and the wound was healed. For a few months subsequently the patient suffered occasional pain in the part, which was always relieved by the prone position. At the end of twelve months she was quite recovered, and has continued, and is at the present date (twelve years having elapsed) perfectly well, the only alteration being, that the bowels do not act without the habitual aid of some simple medicine.

\section{APPENDIX.}

Several questions having been put to me by members of the profession in relation to this interesting case, $I$ have endeavoured, for the sake of conciseness, to comprise the most practical points under the three following forms of inquiry :

J.st. Did the recovery from the fæcal fistula depend on the subsequent treatment adopted? and was it an instance of post hoc propter hoc? 
2nd. Why was the prone position on the face recommended?

3rd. With what object were cubebs prescribed?

In explanation, I reply to the above seriatim-

1st. Quite recently, in conversation with Sir Benjamin Brodie on this subject, he told me that since the above case he has had another similar one of cæcal fistula under his care, in which precisely the same treatment recommended by him proved successful and curative.

2nd. In all those cases of rupture of the cæcum followed by abscess, which Sir Benjamin Brodie has had the opportunity of examining after death, on dissection, the opening has been uniformly at the posterior part of the intestine; consequently, the object of the patient, maintaining the prone position on the face, becomes obvious.

3rd. The use of powdered cubebs pepper was suggested from it having been found to be often very beneficial in cases of internal piles, and especially useful when the patient suffers from hæmorrhage in consequence. The powdered cubebs pepper seems to act by mixing with the fæces, and becoming a topical application to the mucous membrane of the bowel. In the case related by me, the grains of pepper were daily recognisable in the fæcal matter passing through the fistulous opening. The medicine appeared to be grateful to the stomach, and to aid the action of the bowels. 